\title{
A Comparison of Boltzmann and Multigroup Flux-Limited Diffusion Neutrino Transport During the Postbounce Shock Reheating Phase in Core Collapse Supernovae
}

O. E. B. Messer ${ }^{1,2}$, A. Mezzacappa ${ }^{1,2}$, S. W. Bruenn ${ }^{3}$, and M. W. Guidry ${ }^{1,2}$

Submitted for publication in The Astrophysical Journal

\footnotetext{
${ }^{1}$ Physics Division, Oak Ridge National Laboratory, Oak Ridge, TN 37831-6354

${ }^{2}$ Department of Physics and Astronomy, University of Tennessee, Knoxville, TN 37996-1200

${ }^{3}$ Department of Physics, Florida Atlantic University, Boca Raton, FL 33431-0991
} 


\begin{abstract}
We compare Newtonian three-flavor multigroup Boltzmann (MGBT) and (Bruenn's) multigroup flux-limited diffusion (MGFLD) neutrino transport in postbounce core collapse supernova environments. We focus our study on quantities central to the postbounce neutrino heating mechanism for reviving the stalled shock. Stationary-state three-flavor neutrino distributions are developed in thermally and hydrodynamically frozen time slices obtained from core collapse and bounce simulations that implement Lagrangian hydrodynamics and MGFLD neutrino transport. We obtain distributions for time slices at $106 \mathrm{~ms}$ and $233 \mathrm{~ms}$ after core bounce for the core of a $15 \mathrm{M}_{\odot}$ progenitor, and at $156 \mathrm{~ms}$ after core bounce for a $25 \mathrm{M}_{\odot}$ progenitor. For both transport methods, the electron neutrino and antineutrino luminosities, RMS energies, and mean inverse flux factors, all of which enter the neutrino heating rates, are computed as functions of radius and compared. The net neutrino heating rates are also computed as functions of radius and compared.

Notably, we find significant differences in neutrino luminosities and mean inverse flux factors between the two transport methods for both precollapse models and for all three time slices. In each case, the luminosities for each transport method begin to diverge above the neutrinospheres, where the MGBT luminosities become larger than their MGFLD counterparts, finally settling to a constant difference maintained to the edge of the core. We find that the mean inverse flux factors, which describe the degree of forward peaking in the neutrino radiation field, also differ significantly between the two transport methods, with MGBT providing more isotropic radiation fields in the gain region.

Most important, we find, for a region above the gain radius, net heating rates for MGBT that are as much as $\sim 2$ times the corresponding MGFLD rates, and net cooling rates below the gain radius that are typically $\sim 0.8$ times the MGFLD rates. These differences stem from differences in the neutrino luminosities and mean inverse flux factors, which can be as much as $11 \%$ and $24 \%$, respectively. They are greatest at earlier postbounce times for a given progenitor mass and, for a given postbounce time, greater for greater progenitor mass. We discuss the ramifications these new results have for the supernova mechanism.
\end{abstract}

Subject headings: (stars:) supernovae: general 


\section{Introduction}

Ascertaining the core collapse supernova mechanism is a long-standing problem in astrophysics. The current paradigm begins with the collapse of a massive star's iron core and the generation of an outwardly propagating shock wave that results from core rebound. Because of nuclear dissociation and neutrino losses, the shock stagnates. This sets the stage for a shock reheating mechanism whereby neutrino energy deposition via electron neutrino and antineutrino absorption on nucleons behind the shock reenergizes it (Bethe \& Wilson 1985; Wilson 1985).

The shock reheating phase is essential to the supernova's success, but it is precisely this phase that is difficult to simulate realistically. During shock reheating, core electron neutrinos and antineutrinos are radiated from their respective neutrinospheres, and a small fraction of this radiated energy is absorbed in the exterior shocked mantle. The shock reheating depends sensitively on the electron neutrino and antineutrino luminosities, spectra (best characterized by the RMS energies), and angular distributions in the region behind the shock (e.g., see Burrows \& Goshy 1993, Janka \& Müller 1996, Mezzacappa et al. 1998b). These, in turn, depend on the neutrino transport in the semitransparent region encompassing the neutrinospheres, necessitating a neutrino transport treatment that is able to transit accurately and seamlessly between neutrino-thick and neutrino-thin regions.

Various neutrino transport approximations have been implemented in simulating core collapse supernovae. The most sophisticated approximation, which naturally has been used in detailed one-dimensional simulations, is multigroup flux-limited diffusion (MGFLD; e.g., Bowers \& Wilson 1982, Bruenn 1985, Myra et al. 1987). MGFLD closes the neutrino radiation hydrodynamics hierarchy of equations at the level of the first moment (the neutrino flux) by imposing a relationship between the flux and the gradient of the neutrino energy density (the zeroth moment). For example,

$$
\begin{gathered}
F_{\nu}=-\frac{c \Lambda}{3} \frac{\partial U_{\nu}}{\partial r}+\ldots, \\
\Lambda=\frac{1}{1 / \lambda+\left|\partial U_{\nu} / \partial r\right| / 3 U_{\nu}},
\end{gathered}
$$

where $\lambda$ is the neutrino mean free path, and $U_{\nu}$ and $F_{\nu}$ are the neutrino energy density and flux (Bruenn 1985). [Other forms for the flux-limiter $\Lambda$ can be found in Bowers \& Wilson (1982), Levermore \& Pomraning (1981), and Myra et al (1987).] Whereas the limits $\lambda \rightarrow 0$ and $\lambda \rightarrow \infty$ produce the correct diffusion and free streaming fluxes, it is in the critical intermediate regime where the MGFLD approximation is of unknown accuracy. Unfortunately, the quantities central to the postshock neutrino heating mentioned earlier are determined in this regime, and given the sensitivity of the neutrino heating to these quantities it becomes necessary to consider more accurate transport schemes. Moreover, in detailed one-dimensional simulations that have 
implemented elaborate MGFLD neutrino transport (e.g., see Bruenn 1993, Wilson \& Mayle 1993, and Swesty \& Lattimer 1994), explosions were not obtained unless the neutrino heating was boosted by additional phenomena, such as convection. This leaves us with at least two possibilities to consider: (1) Failures to produce explosions in the absence of additional phenomena, such as convection, have resulted from inexact neutrino transport. (2) Additional phenomena may be essential in obtaining explosions.

Option (1) requires further comment. All investigators agree convection will occur during the shock reheating, explosion initiation phase in core collapse supernovae (Herant, Benz, \& Colgate 1992; Miller et al. 1993; Herant et al. 1994; Burrows et al. 1995, Janka \& Müller 1996, Mezzacappa et al. 1998b). Therefore, strictly speaking, all investigators agree the flow will not be spherically symmetric. However, although convection will certainly occur, it may play no significant role in initiating the explosion. It is with this distinction in mind that option (1) need be considered. For example, significant neutrino-driven convection was seen in recent multidimensional simulations employing one-dimensional MGFLD neutrino transport (Mezzacappa et al. 1998b); however, the angle-averaged shock radius, among other quantities, did not differ significantly from its one-dimensional counterpart, and no explosion was obtained.

Ultimately, any successful model of core collapse supernovae will have to reproduce observables that clearly do not originate from spherically symmetric explosions, the most obvious of which is neutron star kicks. At this point, whether or not these kicks are generated during the initiation of the explosion or shortly thereafter is an open question. Note in this regard that simulations that have invoked multidimensional effects such as convection to explain such kicks have had difficulty generating, for example, adequate kick velocities. Mechanisms invoking convection, or aspherical neutrino emission resulting from convection, have not been able to produce kicks in excess of about $300 \mathrm{~km} / \mathrm{s}$, which therefore cannot account for the fastest pulsars - for example, PSR 2224+65, which has a velocity around $800 \mathrm{~km} / \mathrm{s}$ - (Janka \& Müller 1994; Burrows \& Hayes 1995, 1996). Moreover, definitive predictions of neutron star kick velocities from aspherical supernovae will require three-dimensional simulations. (The aforementioned simulations were carried out in two dimensions.) Recent simulations of neutrino-driven convection in two and three dimensions demonstrate that, as expected, longer-wavelength modes break up in three dimensions, rendering the angle-averaged flow qualitatively much closer to spherically symmetric (Knerr et al. 1998; see also Müller 1993 and Janka and Müller 1996). In light of this, it is difficult to see how the already low values for neutron star kick velocities obtained by invoking convection and/or convection-induced anisotropic neutrino emission during the explosion itself could be enhanced when these same simulations are carried out in three dimensions.

In this paper, we compare three-flavor multigroup Boltzmann neutrino transport (MGBT) and (Bruenn's) MGFLD in spherically symmetric, hydrostatic, thermally frozen, postbounce profiles, with an eye toward quantities central to the postbounce neutrino heating mechanism for reviving the stalled shock. In particular, for both transport methods, we compute and compare the neutrino luminosities, RMS energies, mean inverse flux factors, and net heating rates as functions 
of radius, time, and precollapse model. We then discuss the ramifications our results have for the supernova mechanism. This work is a continuation and extension of core collapse simulations (Mezzacappa and Matzner 1989, Mezzacappa and Bruenn 1993a,b,c), in which exact Boltzmann neutrino transport and multigroup flux-limited diffusion were compared.

\section{Initial Models, Codes, and Methodology}

We begin with $15 \mathrm{M}_{\odot}$ and $25 \mathrm{M}_{\odot}$ precollapse models $\mathrm{S} 15 \mathrm{~s} 7 \mathrm{~b}$ and $\mathrm{S} 25 \mathrm{~s} 7 \mathrm{~b}$ provided by Woosley (1995). The initial models were evolved through core collapse and bounce using one-dimensional Lagrangian hydrodynamics and MGFLD neutrino transport coupled to the Lattimer-Swesty equation of state (Lattimer \& Swesty 1991). The data at $106 \mathrm{~ms}$ and $233 \mathrm{~ms}$ after bounce for S15s7b and $156 \mathrm{~ms}$ after bounce for S25s7b were thermally and hydrodynamically frozen. Stationary-state neutrino distributions were computed for these profiles using both MGBT and MGFLD.

The MGBT simulations were performed using BOLTZTRAN: a Newtonian gravity, $O(v / c)$, three-flavor, Boltzmann neutrino transport code developed for the supernova problem and used thus far for studies of stellar core collapse (Mezzacappa \& Matzner 1989, Mezzacappa \& Bruenn 1993abc). The MGFLD simulations were performed using MGFLD-TRANS: a Newtonian gravity,

$O(v / c)$, three-flavor, MGFLD neutrino transport code, which has been used for both core collapse and postbounce evolution (e.g., Bruenn 1985, 1993).

The MGBT simulations used 110 nonuniform spatial zones spanning radii from the origin to $4744 \mathrm{~km}$ and $4673 \mathrm{~km}$ for model S15s7b at $t_{\mathrm{pb}}=106 \mathrm{~ms}$ and $233 \mathrm{~ms}$ respectively, and to 2096 $\mathrm{km}$ for model $\mathrm{S} 25 \mathrm{~s} 7 \mathrm{~b}$ at $t_{\mathrm{pb}}=156 \mathrm{~ms}$. Twelve energy zones spanning a range between 5 and 225 $\mathrm{MeV}$ were used to resolve the neutrino spectra. The MGFLD used the same spatial and energy grids. Simulations with 20 energy zones spanning the same energy range were performed with BOLTZTRAN; no changes in the results presented here were seen.

For the MGBT simulations there is an added dimension: neutrino direction cosine. Because MGBT computes the neutrino distributions as a function of direction cosine and energy for each spatial zone, the isotropy of the neutrino radiation field as a function of radius and neutrino energy is computed from first principles. This is one of the key features distinguishing MGBT and MGFLD. Because the isotropy of the neutrino radiation field is critical to the shock reheating/revival, four Gaussian quadrature sets $\left(2-, 4^{-}, 6^{-}\right.$, and 8-point) were implemented in the MGBT simulations to ensure numerical convergence of the results. 


\section{Results}

For electron neutrino and antineutrino absorption on neutrons and protons, the neutrino heating rate (in $\mathrm{MeV} /$ nucleon) in the region between the neutrinospheres and the shock can be written as

$$
\dot{\epsilon}=\frac{X_{n}}{\lambda_{0}^{a}} \frac{L_{\nu_{\mathrm{e}}}}{4 \pi r^{2}}<E_{\nu_{\mathrm{e}}}^{2}><\frac{1}{\mathrm{~F}}>+\frac{X_{p}}{\bar{\lambda}_{0}^{a}} \frac{L_{\bar{\nu}_{\mathrm{e}}}}{4 \pi r^{2}}<E_{\bar{\nu}_{\mathrm{e}}}^{2}><\frac{1}{\overline{\mathrm{F}}}>
$$

where: $X_{n, p}$ are the neutron and proton fractions; $\lambda_{0}^{a}=\bar{\lambda}_{0}^{a}=G_{F}^{2} \rho\left(g_{V}^{2}+3 g_{A}^{2}\right) / \pi(h c)^{4} m_{B}$; $G_{F} /(\hbar c)^{3}=1.166 \times 10^{-5} \mathrm{GeV}^{-2}$ is the Fermi coupling constant; $\rho$ is the matter density; $g_{V}=1.0$, $g_{A}=1.23 ; m_{B}$ is the baryon mass; and $L_{\nu_{\mathrm{e}}, \bar{\nu}_{\mathrm{e}}},\left\langle E_{\nu_{\mathrm{e}}, \bar{\nu}_{\mathrm{e}}}^{2}\right\rangle$, and $\mathrm{F}, \overline{\mathrm{F}}$ are the electron neutrino and antineutrino luminosities, RMS energies, and mean inverse flux factors, defined by

$$
\begin{gathered}
L_{\nu_{\mathrm{e}}}=4 \pi r^{2} \frac{2 \pi c}{(h c)^{3}} \int d E_{\nu_{\mathrm{e}}} d \mu_{\nu_{\mathrm{e}}} E_{\nu_{\mathrm{e}}}^{3} \mu_{\nu_{\mathrm{e}}} f, \\
\left\langle E_{\nu_{\mathrm{e}}}^{2}\right\rangle=\frac{\int d E_{\nu_{\mathrm{e}}} d \mu_{\nu_{\mathrm{e}}} E_{\nu_{\mathrm{e}}}^{5} f}{\int d E_{\nu_{\mathrm{e}}} d \mu_{\nu_{\mathrm{e}}} E_{\nu_{\mathrm{e}}}^{3} f}, \\
\left\langle\frac{1}{\mathrm{~F}}\right\rangle=\frac{\int d E_{\nu_{\mathrm{e}}} d \mu_{\nu_{\mathrm{e}}} E_{\nu_{\mathrm{e}}}^{3} f}{\int d E_{\nu_{\mathrm{e}}} d \mu_{\nu_{\mathrm{e}}} E_{\nu_{\mathrm{e}}}^{3} \mu_{\nu_{\mathrm{e}}} f}=\frac{c U_{\nu_{\mathrm{e}}}}{F_{\nu_{\mathrm{e}}}} .
\end{gathered}
$$

In equations (4)-(6),$f$ is the electron neutrino distribution function, which is a function of the electron neutrino direction cosine, $\mu_{\nu_{\mathrm{e}}}$, and energy, $E_{\nu_{\mathrm{e}}}$. In equation (6), $U_{\nu_{\mathrm{e}}}$ and $F_{\nu_{\mathrm{e}}}$ are the electron neutrino energy density and flux. Corresponding quantities can be defined for the electron antineutrinos. Success or failure to generate explosions via neutrino reheating must ultimately rest on the three quantities defined in equations (4)-(6). Both the MGBT and the MGFLD stationary state distributions were computed in the same thermally and hydrodynamically frozen matter configuration.

In Figure 1, at $233 \mathrm{~ms}$ after bounce for model S15s7b, we plot the electron neutrino and antineutrino RMS energies, luminosities, and mean inverse flux factors as functions of radius for our (8-point Gaussian quadrature) MGBT and MGFLD runs. Energy-averaged electron neutrinoand antineutrino-spheres were located by calculating an energy-integrated neutrino depth defined by

$$
\bar{\tau}=\frac{\int_{\infty}^{r} d r^{\prime} \int_{0}^{\infty} d E_{\nu} d \mu_{\nu} E_{\nu}^{3} f / \lambda}{\int_{0}^{\infty} d E_{\nu} d \mu_{\nu} E_{\nu}^{3} f}
$$


and determining the radius at which $\bar{\tau}=2 / 3$. The neutrinospheres (at $57 \mathrm{~km}$ and $48 \mathrm{~km}$, for electron neutrinos and antineutrinos, respectively), and the location of the shock (at $191 \mathrm{~km}$ ), are indicated by arrows. The gain radius (neutrino-energy integrated), located at $98 \mathrm{~km}$, is also marked by an arrow. For the electron neutrinos, the differences in RMS energies between MGBT and MGFLD are at most $2 \%$ throughout most of the region plotted, although MGBT consistently gives higher energies. The differences between MGBT and MGFLD antineutrino RMS energies are smaller, and neither transport scheme yields consistently higher values. It should be noted that we expect larger differences when a fully hydrodynamic simulation is carried out, with MGBT giving harder spectra (Mezzacappa and Bruenn 1993a,c; see also Burrows 1998). In a static matter configuration, differences that result from different treatments of the neutrino energy shift measured by comoving observers do not occur.

Significant differences between MGBT and MGFLD are evident when comparing the neutrino and antineutrino luminosities and mean inverse flux factors. The luminosity curves for both electron neutrinos and antineutrinos coincide for both transport methods until the neutrinospheres are approached from below. Just below the neutrinospheres, the MGBT luminosities diverge upward from the MGFLD luminosities, differing by $7 \%$ (4\% for antineutrinos) at the neutrinospheres. The root cause of this difference is that the MGFLD interpolation underestimates the flux in this region. After a decline from this maximum difference, the fractional difference grows from approximately $3 \%$ at the base of the gain region to a constant difference of $6 \%$ beyond about $170 \mathrm{~km}$. Similar behavior is exhibited by the antineutrino luminosities, with the same fractional differences, $3 \%$ and $6 \%$, seen at the base of the gain region and near the shock, respectively.

For the electron neutrinos, the fractional difference between $<1 / \mathrm{F}>_{\text {MGFLD }}$ and $<1 / \mathrm{F}>_{\text {MGBT }}$ is $2 \%, 8 \%$, and $12 \%$ at the neutrinosphere, gain radius, and shock, respectively. Just above the shock, the difference converges to $10 \%$, and is maintained to the edge of the core. Focusing on the semitransparent region, $<1 / F>_{M G F L D}$ is greater until the gain radius is approached from below; i.e., the MGFLD neutrino radiation field is more isotropic than the MGBT radiation field below these radii. At $80 \mathrm{~km}$, as the gain radius is approached, MGFLD computes a sharp decrease in $<1 / F>$. Looking at Figure 2, where we plot the density and the sum of the MGBT electron neutrino and antineutrino luminosities for this time slice as functions of radius, it is evident as the gain radius is approached from below that the luminosity sum begins to turn over, marking the enclosure of the neutrino and antineutrino source. Therefore, for MGFLD, the accelerated transition to free streaming occurs not at the neutrinospheres, as might have been anticipated, but at a radius within which most of the neutrino and antineutrino source is enclosed. For example, for this time slice the electron neutrino luminosity at the neutrinosphere is only $76 \%$ of its peak value.

In Figure 1, there is a second dip and a small spike in $<1 / F>_{\text {MGFLD }}$ at $106 \mathrm{~km}$ and $163 \mathrm{~km}$, respectively; $<1 / \mathrm{F}>_{\mathrm{MGBT}}$ is smooth through these radii. Again, examining Figure 2, the density shows discontinuities at $106 \mathrm{~km}$ and $163 \mathrm{~km}$, which produce these features. The density profile flattens at $106 \mathrm{~km}$ and then drops precipitously. There is a corresponding flattening and sharp 
drop in $<1 / F>_{\text {MGFLD }}$ in this region. The density actually increases at $160 \mathrm{~km}$, then immediately falls off. This results in an increase in $<1 / F>_{\text {MGFLD }}$ at that radius, followed by a sharp decrease. In both cases, the isotropy of the MGFLD neutrino radiation field is altered by local conditions.

For the electron antineutrinos, the same features are seen in $<1 / F>_{\text {MGFLD }}$. The fractional difference is $0 \%, 11 \%$, and $11 \%$ at the neutrinosphere, gain radius, and shock, respectively. The initial sharp decrease in $<1 / F>_{\text {MGFLD }}$ occurs at a smaller radius. The antineutrino luminosity maximum, i.e., the point at which the antineutrino source is enclosed, is at a smaller radius.

In Figure 3, we plot the same three quantities for the earlier time slice in our $15 \mathrm{M}_{\odot}$ model $\left(t_{\mathrm{pb}}=106 \mathrm{~ms}\right)$. The differences between MGBT and MGFLD are similar to those seen at the later postbounce time $\left(t_{\mathrm{pb}}=233 \mathrm{~ms}\right)$. The electron neutrino RMS energies are slightly higher for MGBT, again by about $2 \%$. The differences in antineutrino RMS energies are again variable in both sign and magnitude, but are never more than $2 \%$. The difference in luminosity is $11 \%$ ( $8 \%$ for antineutrinos) at the neutrinosphere, $11 \%$ ( $7 \%$ for antineutrinos) at the gain radius, and settles to a constant difference of $9 \%$ (6\% for antineutrinos) above $170 \mathrm{~km}$. As in the later time slice, MGFLD underestimates the flux beginning below the neutrinospheres, extending everywhere above the neutrinospheres, which in turn results in a lower luminosity.

For electron neutrinos, the fractional difference between $<1 / \mathrm{F}>_{\text {MGFLD }}$ and $<1 / \mathrm{F}>_{\text {MGBT }}$ is $4 \%, 2 \%$, and $17 \%$ at the neutrinosphere, gain radius, and shock, respectively. Most important, the same abrupt decrease in $<1 / F>_{\text {MGFLD }}$ beginning just above the gain radius is evident. In Figure 4, we plot the density and the sum of the MGBT electron neutrino and antineutrino luminosities for $t_{\mathrm{pb}}=106 \mathrm{~ms}$. The decrease in $<1 / \mathrm{F}>_{\text {MGFLD }}$ again occurs near the radius where the luminosity sum turns over: $128 \mathrm{~km}$. There is also a small dip in $<1 / \mathrm{F}>_{\text {MGFLD }}$ at $172 \mathrm{~km}$. Looking at Figure 4, the only significant dip in density occurs at $172 \mathrm{~km}$, causing a local decrease in the isotropy of the MGFLD radiation field.

For the electron antineutrinos, the difference between $<1 / \mathrm{F}>_{\text {MGFLD }}$ and $<1 / \mathrm{F}>_{\text {MGBT }}$ is $3 \%, 2 \%$, and $16 \%$ at the neutrinosphere, gain radius, and shock, respectively. The point at which $<1 / \mathrm{F}>_{\text {MGFLD }}$ drops below $<1 / F>_{\text {MGBT }}$ is translated inwards, as expected, towards the antineutrino luminosity maximum. For both electron neutrinos and antineutrinos, a difference of about $13 \%$ is maintained with increasing radius above the shock.

Figure 5 contains the same information as Figures 1 and 3, but for our $25 \mathrm{M}_{\odot}$ model at $156 \mathrm{~ms}$ after bounce. The similarities between the $25 \mathrm{M}_{\odot}$ and the $15 \mathrm{M}_{\odot}$ results are striking, considering the marked difference in core structure. The identical trend in RMS energies is again seen: there are small differences for the electron neutrinos, but consistently higher values are obtained with MGBT (again, $\sim 2 \%$ higher); there are smaller, sometimes oscillating, differences between MGBT and MGFLD for the electron antineutrinos. The absolute value of the neutrino luminosities at the gain radius is almost a factor of 2 greater than in either of the $15 \mathrm{M}_{\odot}$ slices, yet the relative differences between the luminosities computed by the two transport methods are similar. The electron neutrino luminosities differ by $9 \%$ (5\% for antineutrinos) at the neutrinosphere, $11 \%(9 \%$ 
for antineutrinos) at the gain radius, and 9\% (7\% for antineutrinos) at the shock. The difference at the shock is maintained to the edge of the core. Again, this difference in luminosity is caused by an underestimation of the flux by MGFLD.

Among the three slices considered, the $25 \mathrm{M}_{\odot}$ core gives rise to the most dramatic differences in $\langle 1 / F>$. For electron neutrinos, we find fractional differences of $1 \%, 2 \%$, and $24 \%$ in $<1 / \mathrm{F}>$ at the neutrinosphere, gain radius, and shock, respectively. There is a sharp decrease in $<1 / \mathrm{F}>_{\text {MGFLD }}$ at $109 \mathrm{~km}$. As in the $15 \mathrm{M}_{\odot}$ case, this change in $<1 / \mathrm{F}>_{\text {MGFLD }}$ is correlated with the enclosure of the neutrino source. This correlation is evident in Figure 6, where we plot the density and sum of the MGBT electron neutrino and antineutrino luminosities for this time slice. Note that the MGBT luminosity sum begins to turn over near $109 \mathrm{~km}$, coincident with the sharp decrease in $<1 / F>_{\text {MGFLD }}$.

There are other precipitous drops in $<1 / F>_{\text {MGFLD }}$ at $125 \mathrm{~km}$ and $162 \mathrm{~km}$. Also apparent in Figure 6 are precipitous drops in density at these radii. These drops in density produce changes in the local neutrino radiation field computed by MGFLD.

For electron antineutrinos, $<1 / F>_{\text {MGFLD }}$ exhibits similar structure, with fractional differences of $0 \%, 1 \%$, and $19 \%$ at the neutrinosphere, gain radius, and shock, respectively. $<1 / \mathrm{F}>_{\text {MGFLD }}$ for antineutrinos also contains three dips: $<1 / \mathrm{F}>_{\text {MGFLD }}$ drops below $<1 / \mathrm{F}>_{\text {MGBT }}$ at $105 \mathrm{~km}$, where most of the antineutrino source is enclosed, and additional dips at $125 \mathrm{~km}$ and $162 \mathrm{~km}$ are also evident, again resulting from the density dips visible in Figure 6. For both electron neutrinos and antineutrinos, constant differences $\sim 12 \%$ are seen above the shock.

Because each of the quantities plotted in Figures 1, 3, and 5 is consistently greater for MGBT (while this is not strictly true for the antineutrino RMS energies in our stationary state comparisons, in a fully dynamical simulation these energies will be consistently higher for MGBT [Mezzacappa and Bruenn 1993a,c; see also Burrows 1998]), and because the neutrino heating rate is proportional to each of them, MGBT yields a significantly higher heating rate. As an example, just above the gain radius for model S15s7b at $t_{\mathrm{pb}}=233 \mathrm{~ms}$, at the point of peak heating, MGBT yields a heating rate from neutrino absorption that is $(102 \%)^{2} \times 110 \% \times 112 \%$ of the MGFLD rate. Note in equation (3) that the heating rate depends linearly on both the neutrino luminosity and the mean inverse flux factor. A reliable determination of the heating rate in and around the gain region therefore depends on a realistic solution of the transport equation in which both of these quantities are determined self consistently.

In Figure 7, for MGBT and MGFLD, we plot the net neutrino heating rates as functions of radius for model $\mathrm{S} 15 \mathrm{~s} 7 \mathrm{~b}$ at $t_{\mathrm{pb}}=233 \mathrm{~ms}$. (As described in Section 2, the results from four Gaussian quadrature sets are plotted to demonstrate numerical convergence.) These rates include the contributions from both the electron neutrinos and antineutrinos, and were computed using the following formulae: 


$$
(d \epsilon / d t)_{i}=c \int E_{\nu}^{3} d E_{\nu}\left[\psi_{i}^{0} / \lambda_{i}^{(a)}-j_{i}\left(1-\psi_{i}^{0}\right)\right] / \rho(h c)^{3}
$$

where $\epsilon$ is the internal energy per gram; $E_{\nu}, \psi_{i}^{0}, \lambda_{i}^{(a)}$, and $j_{i}$ are the electron neutrino or antineutrino energy, zeroth angular moment, absorption mean free path, and emissivity, respectively; $i=1$ corresponds to electron neutrinos, and $i=2$ corresponds to electron antineutrinos. Only the contributions from neutrino emission and absorption were included: in our models, contributions from neutrino-electron scattering and other processes contribute only a few percent to the neutrino heating rate at and before $233 \mathrm{~ms}$ after bounce; they become more important $(\sim 15 \%)$ at later times. The (8-point Gaussian quadrature) MGBT simulation yields a net heating rate just above the gain radius that is $\sim 1.3$ times the MGFLD rate, and a net cooling rate below the gain radius that is consistently $\sim 0.8$ times the MGFLD rate. Comparing the net heating rate to the luminosity sum in Figure 2, the extent of the gain region (from $98 \mathrm{~km}$ to $180 \mathrm{~km}$ ) is well correlated with the region between the luminosity maximum and the radius where the luminosity levels off to a constant value (from $101 \mathrm{~km}$ to $170 \mathrm{~km}$ ). Note also that the gain radius is located at a smaller radius for MGBT, and consequently, the size of the net heating region below the shock is somewhat larger.

For (8-point Gaussian quadrature) MGBT and MGFLD, Figure 8 shows the net heating curves for $\mathrm{S} 15 \mathrm{~s} 7 \mathrm{~b}$ at an earlier postbounce time, $t_{\mathrm{pb}}=106 \mathrm{~ms}$. The region between the neutrinospheres and the shock is a bit smaller: The shock is approximately at the same radius, but the energy-averaged electron neutrinosphere is closer to the shock (at $84 \mathrm{~km}$, versus $58 \mathrm{~km}$ for $t_{\mathrm{pb}}=233 \mathrm{~ms}$ ). Similar net heating profiles are seen, but the differences between MGBT and MGFLD are even greater. MGBT yields a net heating rate that is $\sim 2$ times the MGFLD rate just above the base of the gain region. Again, the cooling rate is consistently $\sim 0.8$ times the MGFLD rate below the gain radius. The correlation between the gain region and the region between the luminosity-sum maximum and the point at which the luminosity sum is constant is also quite strong for this time slice. The gain radius $(103 \mathrm{~km})$ is just below the luminosity maximum (107 $\mathrm{km})$; also, the luminosity levels off at a radius $(173 \mathrm{~km})$ near the upper edge of the gain region $(182 \mathrm{~km})$.

Figure 9 contains the net heating curves for our $25 \mathrm{M}_{\odot}$ model at $t_{\mathrm{pb}}=156 \mathrm{~ms}$. Again, in this case the (8-point Gaussian quadrature) MGBT net heating rate is $\sim 2$ times the MGFLD rate just above the base of the gain region, and the net cooling rate below the gain radius is consistently $\sim 0.8$ times the MGFLD rate. The gain radius $(111 \mathrm{~km})$ and the luminosity-sum maximum (108 $\mathrm{km}$ ) are strongly correlated, as are the radius marking the upper extent of the gain region (180 $\mathrm{km})$ and the radius at which the luminosity sum levels off $(170 \mathrm{~km})$. Although the differences in the net heating rate are comparable to those seen in the $t_{\mathrm{pb}}=106 \mathrm{~ms}$ slice for model S15s7b, they occur at a significantly later postbounce time. At a given postbounce time, MGBT provides the greatest enhancement in net heating for larger progenitor masses. 


\section{Summary, Discussion, and Conclusions}

Comparing three-flavor MGBT and three-flavor MGFLD in postbounce supernova environments, we find that MGBT leads to a significant increase/decrease in the net heating/cooling rate, particularly above/below the gain radius. The MGBT net heating rate can be as much as $\sim 2$ times the MGFLD net heating rate above the gain radius, with net cooling rates that are typically $\sim 0.8$ times the MGFLD rate below the gain radius. These differences stem primarily from differences in the neutrino luminosities and mean inverse flux factors; the heating rate is linearly proportional to both these quantities, and differences in both add to produce a significant difference in the net heating rate.

In Figure 10, we plot the sum of the electron neutrino and antineutrino luminosities computed in the MGFLD S15s7b dynamic simulation for several different postbounce times. It is important to note that the total luminosity changes by $\sim 5-15 \%$ between $100 \mathrm{~km}$ and $200 \mathrm{~km}$ on time scales $\sim 30 \mathrm{~ms}$. Moreover, the light crossing time between $100 \mathrm{~km}$ and $200 \mathrm{~km}$ is $\sim 1 / 3 \mathrm{~ms}$. Therefore, the neutrino source in our simulations changes on time scales that are two orders of magnitude greater than the time scales on which stationary state is established in this region. This suggests our stationary state results closely reflect what will occur in dynamic simulations.

We also observe that the differences in the net heating rate are greatest at earlier postbounce times for a given progenitor mass, and greater at any given postbounce time for greater progenitor mass. This is illustrated in Table 1. The increase in net heating with increased progenitor mass is advantageous because of the slower fall-off in the preshock accretion ram pressure.

These results have at least two important ramifications for the supernova mechanism:

(1) With the dramatic increase in net heating above the gain radius, which is seen in all of our postbounce slices, it may be possible to obtain explosions in one dimension without multidimensional effects such as convection; this will be aided by the decrease in net cooling below the gain radius. It should be noted that our postbounce slices come from full radiation hydrodynamics simulations implementing MGFLD that marginally failed to produce explosions (Bruenn 1993). The marginality of Bruenn's simulations is an important motivating factor in comparing our MGBT results solely with his MGFLD results. All else being equal, increases in net heating of the magnitude documented here would most likely lead to explosions. However, simulations coupling MGBT and the core hydrodynamics must be carried out in order to compute any feedbacks. It remains to be seen whether the MGBT heating rate will remain sufficiently high to generate an explosion. Also, the effects of using general relativistic gravity, hydrodynamics, and neutrino transport must be explored, especially if explosions are obtained in the Newtonian limit. For example, the redshifted neutrino energies, the smaller gain region, and the greater infall velocities in the gain region will most likely conspire to make explosions more difficult to obtain (Mezzacappa et al. 1998b, DeNisco, Bruenn, \& Mezzacappa 1998, Bruenn, DeNisco, \& Mezzacappa 1998). 
(2) With the dramatic increase in net heating occurring near the base of the gain region, we anticipate that MGBT coupled to two-dimensional hydrodynamics will yield more vigorous neutrino-driven convection than was seen in Mezzacappa et al. (1998b), where two-dimensional hydrodynamics was coupled to one-dimensional MGFLD. The combination of increased net heating and more vigorous neutrino-driven convection would be more favorable for shock revival.

In closing, our results are promising, and their ramifications for core collapse supernovae and, in particular, for the postbounce neutrino-heating, shock-revival mechanism await oneand two-dimensional dynamical simulations with MGBT coupled to the core hydrodynamics. One-dimensional simulations are currently underway, and we plan to report on them soon.

\section{Acknowledgements}

BM was supported at the University of Tennessee under NASA grant NRA-96-04-GSFC-073. $\mathrm{AM}$ and MWG were supported at the Oak Ridge National Laboratory, which is managed by Lockheed Martin Energy Research Corporation under DOE contract DE-AC05-96OR22464, and at the University of Tennessee, under DOE contract DE-FG05-93ER40770. SWB was supported at Florida Atlantic University under NASA grant NRA-96-04-GSFC-073 and NSF grant AST-9618423. The simulations presented in this paper were carried out on the Cray C90 at the National Energy Research Supercomputer Center, the Cray Y/MP at the North Carolina Supercomputer Center, and the Cray Y/MP and Silicon Graphics Power Challenge at the Florida Supercomputer Center. AM and SWB gratefully acknowledge the hospitality of the Institute for Theoretical Physics, Santa Barbara, which is supported in part by the National Science Foundation under grant PHY94-07194. The authors also want to thank the referee, John Hayes, for suggestions that improved the content of this paper. 


\section{References}

Bethe, H. \& Wilson, J. R. 1985, ApJ, 295, 14

Bowers, R. L. \& Wilson, J. R. 1982, ApJS, 50, 115

Brown, G. E., Bruenn, S. W., \& Wheeler, J. C. 1992, Comments Astrophys. 16, 153

Bruenn, S. W. 1985, ApJS, 58, 771

Bruenn, S. W. 1993, in Nuclear Physics in the Universe, eds. M. W. Guidry \& M. R. Strayer (Bristol: IOP Publishing), p. 31

Bruenn, S. W. \& Mezzacappa, A. 1994, ApJ, 433, L45

Bruenn, S. W., DeNisco, K., \& Mezzacappa, A. 1998, ApJ, submitted

Bruenn, S. W. \& Dineva, T. 1996, ApJ, 458, L71

Burrows, A. 1998, in Proceedings of the 18th Texas Symposium on Relativistic Astrophysics, eds. A. Olinto, J. Frieman, \& D. Schramm (Singapore: World Scientific Press), in press

Burrows, A. \& Goshy, J. 1993, ApJ, 416, L75

Burrows, A. \& Hayes, J. 1995, in High-Velocity Neutron Stars and Gamma Ray Bursts, eds. E. Rothschild \& R. E. Lingenfelter (Woodbury, N.Y.: American Institute of Physics), p. 25

Burrows, A. \& Hayes, J. 1996, Phys. Rev. Lett. , 76, 352

Burrows, A., Hayes, J., \& Fryxell, B. A. 1995, ApJ, 450, 830

Chevalier, R. A. 1989, ApJ, 346, 847

DeNisco, K., Bruenn, S. W. \& Mezzacappa, A. 1998, in Second Oak Ridge Symposium on Atomic and Nuclear Astrophysics, ed. A. Mezzacappa (Bristol: IOP Publishing), in press

Dgani, R. \& Janka, H.-Th. 1992, A\& A 256, 428

Herant, M. E., Benz, \& Colgate, S. A. 1992, ApJ, 395, 642

Herant, M. E., Benz, W., Hix, W. R., Fryer, C., \& Colgate, S. A. 1994, ApJ, 435, 339

Janka, H.-Th. 1992, A\& A 256, 452

Janka, H.-Th., \& Müller, E. 1994, A\& A 290, 496

Janka, H.-Th., \& Müller, E. 1996, A\& A 306, 167

Keil, W., Janka, H.-Th., \& Müller, E. 1996, ApJ, 473, L111

Knerr, J. M., Mezzacappa, A., Blondin, J. M., and Bruenn, S. W., ApJ, in preparation

Lattimer, J. M. \& Swesty , F. D. 1991, Nucl. Phys. A, 535, 331 
Levermore, C. D. \& Pomraning, G. C. 1981, ApJ, 248, 321

Mezzacappa, A. \& Matzner, R. A. 1989, ApJ, 343, 853

Mezzacappa, A. \& Bruenn, S. W. 1993a, ApJ, 405, 637

Mezzacappa, A. \& Bruenn, S. W. 1993b, ApJ, 405, 669

Mezzacappa, A. \& Bruenn, S. W. 1993c, ApJ, 410, 710

Mezzacappa, A., Calder, A. C., Bruenn, S. W., Blondin, J. M., Guidry, M. W., Strayer, M. R., \& Umar, A. S. 1998a, ApJ, 493, 848

Mezzacappa, A., Calder, A. C., Bruenn, S. W., Blondin, J. M., Guidry, M. W., Strayer, M. R., \& Umar, A. S. 1998b, ApJ, 495, 911

Müller, E. 1993, in Proceedings of the 7th Workshop on Nuclear Astrophysics, eds. W. Hillebrandt \& E. Müller (Garching: Max-Planck-Institut für Astrophysik), p. 27

Miller, D. S., Wilson, J. R., \& Mayle, R. W. 1993, ApJ, 415, 278

Myra, E., Bludman, S., Hoffman, Y., Lichtenstadt, I., Sack, N., \& Van Riper, K. 1987, ApJ, 318, 744

Swesty, F. D. \& Lattimer, J. M. 1994, ApJ, 425, 195

Wilson, J. R. \& Mayle, R. W. 1993, Phys. Rep., 227, 97

Wilson, J. R. 1985, in Numerical Astrophysics, eds. J. M. Centrella, J. M. LeBlanc, \& R. L. Bowers (Boston: Jones \& Bartlett), p. 422

Woosley, S. E. 1995, private communication 


\section{Figure Captions}

Fig. 1.- At $t_{\mathrm{pb}}=233 \mathrm{~ms}$, for model S15s7b, we plot the electron neutrino and antineutrino RMS energies, luminosities, and mean inverse flux factors versus radius for both Boltzmann neutrino transport and Bruenn's MGFLD. The location of the energy-averaged electron neutrinosphere and anti-neutrinosphere and the locations of the gain radius and shock are indicated by arrows.

Fig. 2.- The density and MGBT electron neutrino and antineutrino luminosity sum are plotted versus radius for model $\mathrm{S} 15 \mathrm{~s} 7 \mathrm{~b}$ at $t_{\mathrm{pb}}=233 \mathrm{~ms}$.

Fig. 3.- At $t_{\mathrm{pb}}=106 \mathrm{~ms}$, for model S15s7b, we plot the electron neutrino and antineutrino RMS energies, luminosities, and mean inverse flux factors versus radius for both Boltzmann neutrino transport and Bruenn's MGFLD. The location of the energy-averaged electron neutrinosphere and anti-neutrinosphere and the location of the gain radius and shock are indicated by arrows.

Fig. 4.- The density and MGBT electron neutrino and antineutrino luminosity sum are plotted versus radius for model $\mathrm{S} 15 \mathrm{~s} 7 \mathrm{~b}$ at $t_{\mathrm{pb}}=106 \mathrm{~ms}$.

Fig. 5.- At $t_{\mathrm{pb}}=156 \mathrm{~ms}$, for model S25s7b, we plot the electron neutrino and antineutrino RMS energies, luminosities, and mean inverse flux factors versus radius for both Boltzmann neutrino transport and Bruenn's MGFLD. The location of the energy-averaged electron neutrinosphere and anti-neutrinosphere and the location of the gain radius and the shock are indicated by arrows.

Fig. 6.- The density and MGBT electron neutrino and antineutrino luminosity sum are plotted versus radius for model $\mathrm{S} 25 \mathrm{~s} 7 \mathrm{~b}$ at $t_{\mathrm{pb}}=156 \mathrm{~ms}$.

Fig. 7.- At $t_{\mathrm{pb}}=233 \mathrm{~ms}$, for model $\mathrm{S} 15 \mathrm{~s} 7 \mathrm{~b}$, the net neutrino heating rates are plotted versus radius for both Boltzmann neutrino transport and MGFLD. The location of the energy-averaged electron neutrinosphere and the location of the shock are indicated by arrows. The results from four Gaussian quadrature sets are plotted to demonstrate numerical convergence.

Fig. 8.- At $t_{\mathrm{pb}}=106 \mathrm{~ms}$, for model S15s7b, the net neutrino heating rates for MGFLD and (8-point Gaussian quadrature) Boltzmann transport are plotted versus radius, along with the locations of the energy-averaged electron neutrinosphere and the shock.

Fig. 9.- At $t_{\mathrm{pb}}=156 \mathrm{~ms}$, for model S25s7b, the net neutrino heating rates for MGFLD and (8-point Gaussian quadrature) Boltzmann transport are plotted versus radius, along with the locations of the energy-averaged electron neutrinosphere and the shock.

Fig. 10. - The sum of the electron neutrino and antineutrino luminosities from the MGFLD dynamic run for model S15s7b are plotted versus radius. The luminosities from five different postbounce times are shown. 


\section{Tables}

Table 1: Maximum Net Heating/Cooling Rates

\begin{tabular}{lccc}
\hline \hline Progenitor Mass $\left[\mathrm{M}_{\odot}\right]$ & $t_{p b}[\mathrm{~ms}]$ & Maximum Net Heating Ratio & Maximum Net Cooling Ratio \\
\hline 15 & 106 & 2.0 & 0.8 \\
& 233 & 1.3 & 0.8 \\
25 & 156 & 2.0 & 0.8 \\
\hline
\end{tabular}




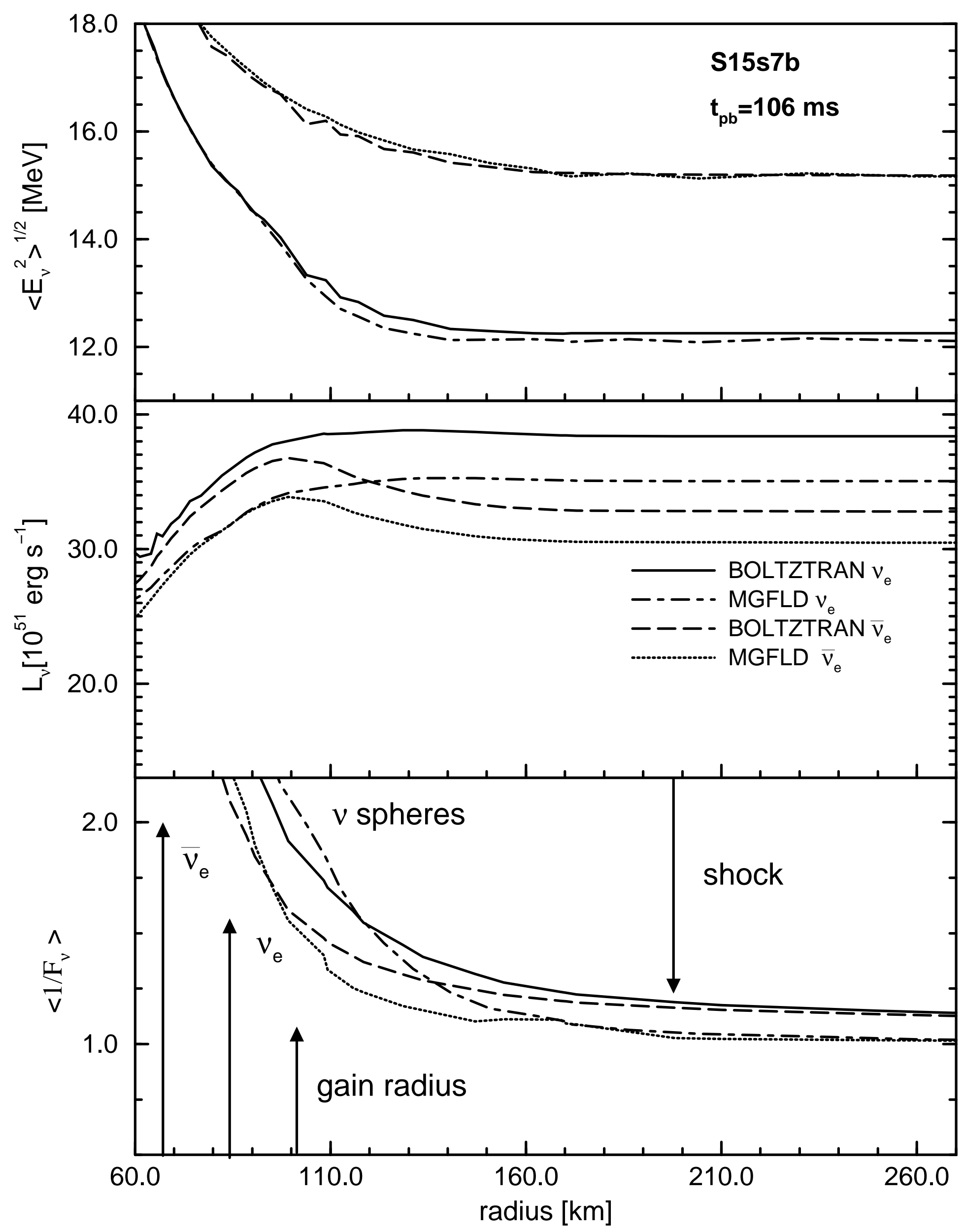

Figure 3 


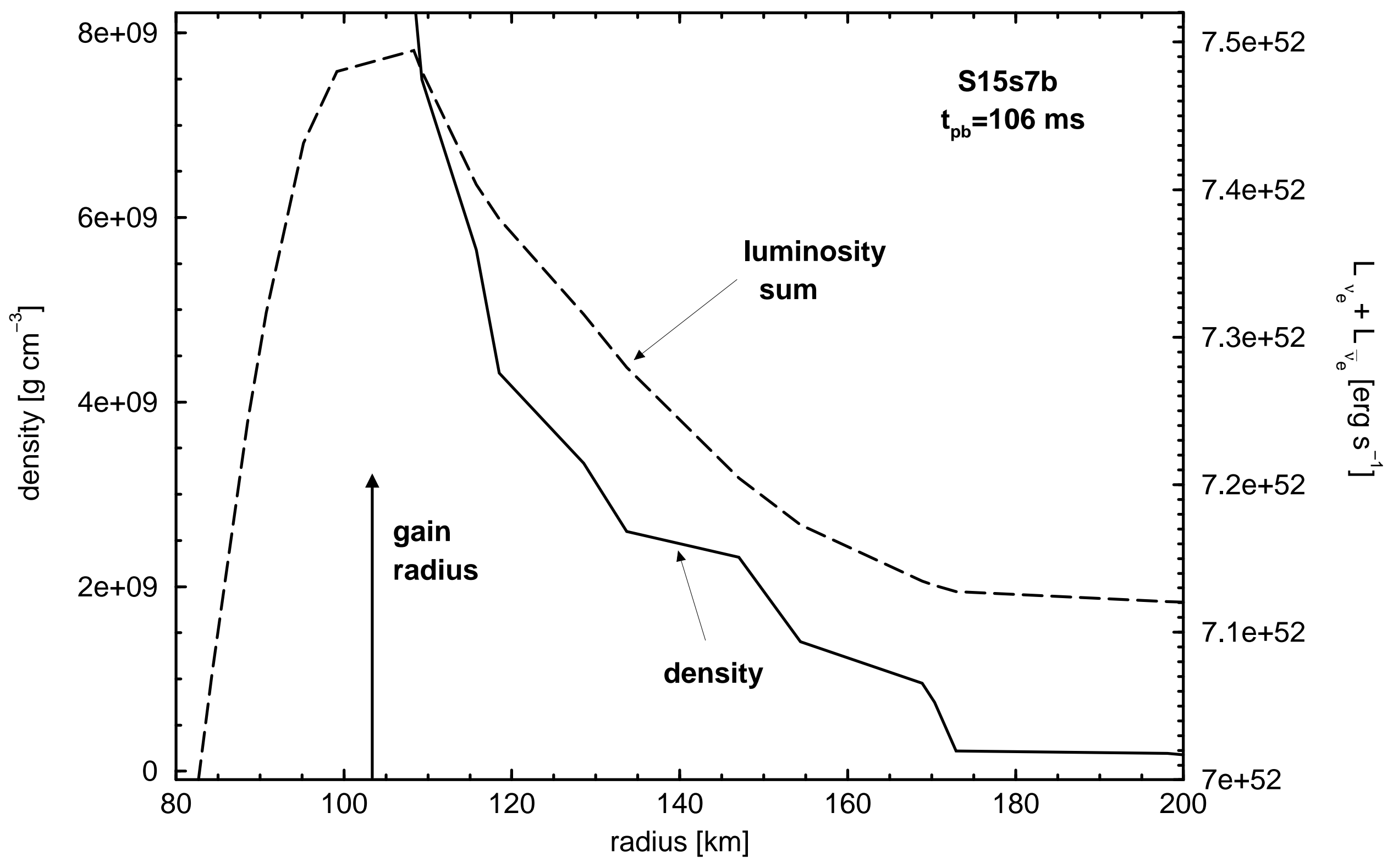

Figure 4 


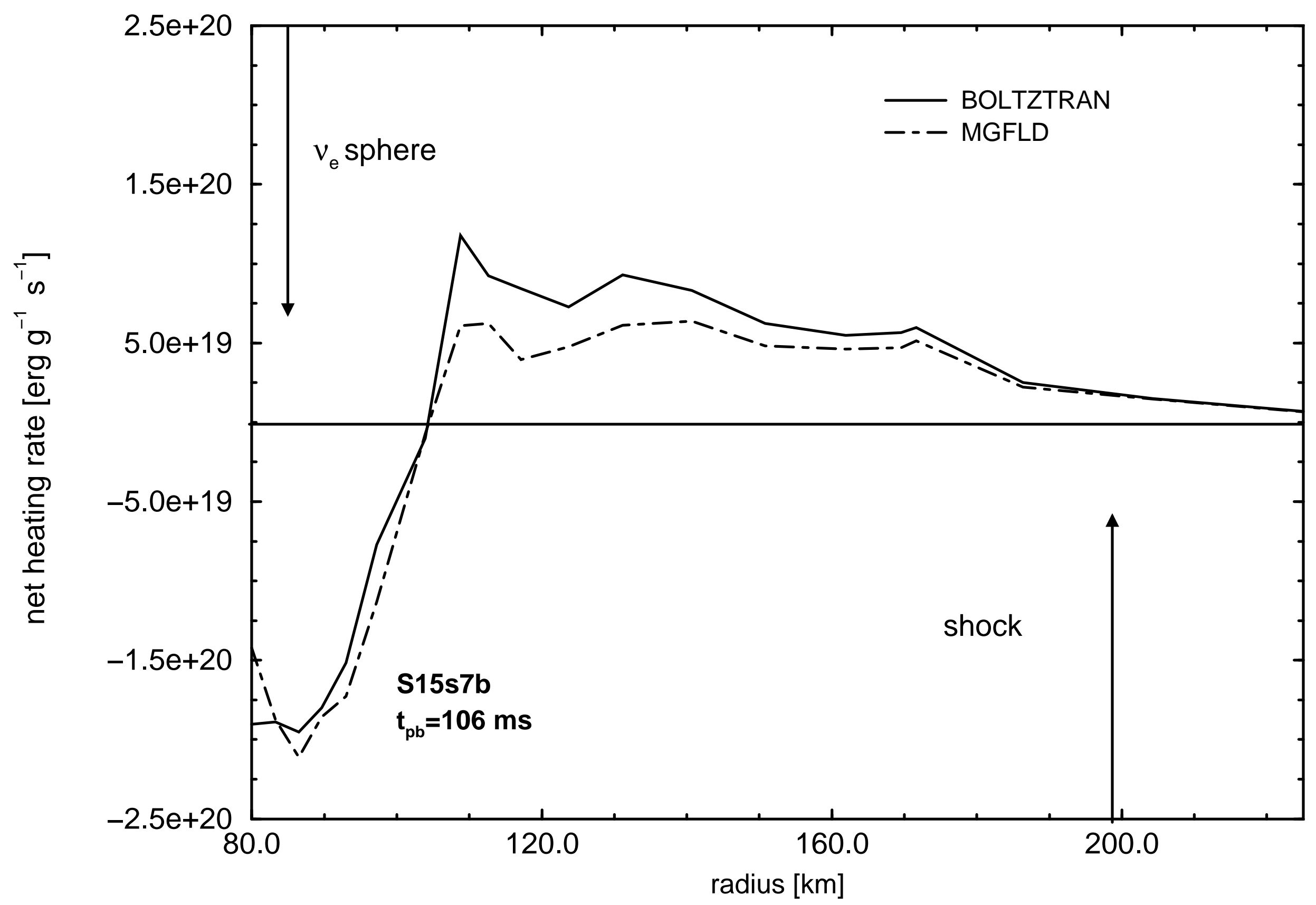

Figure 8 


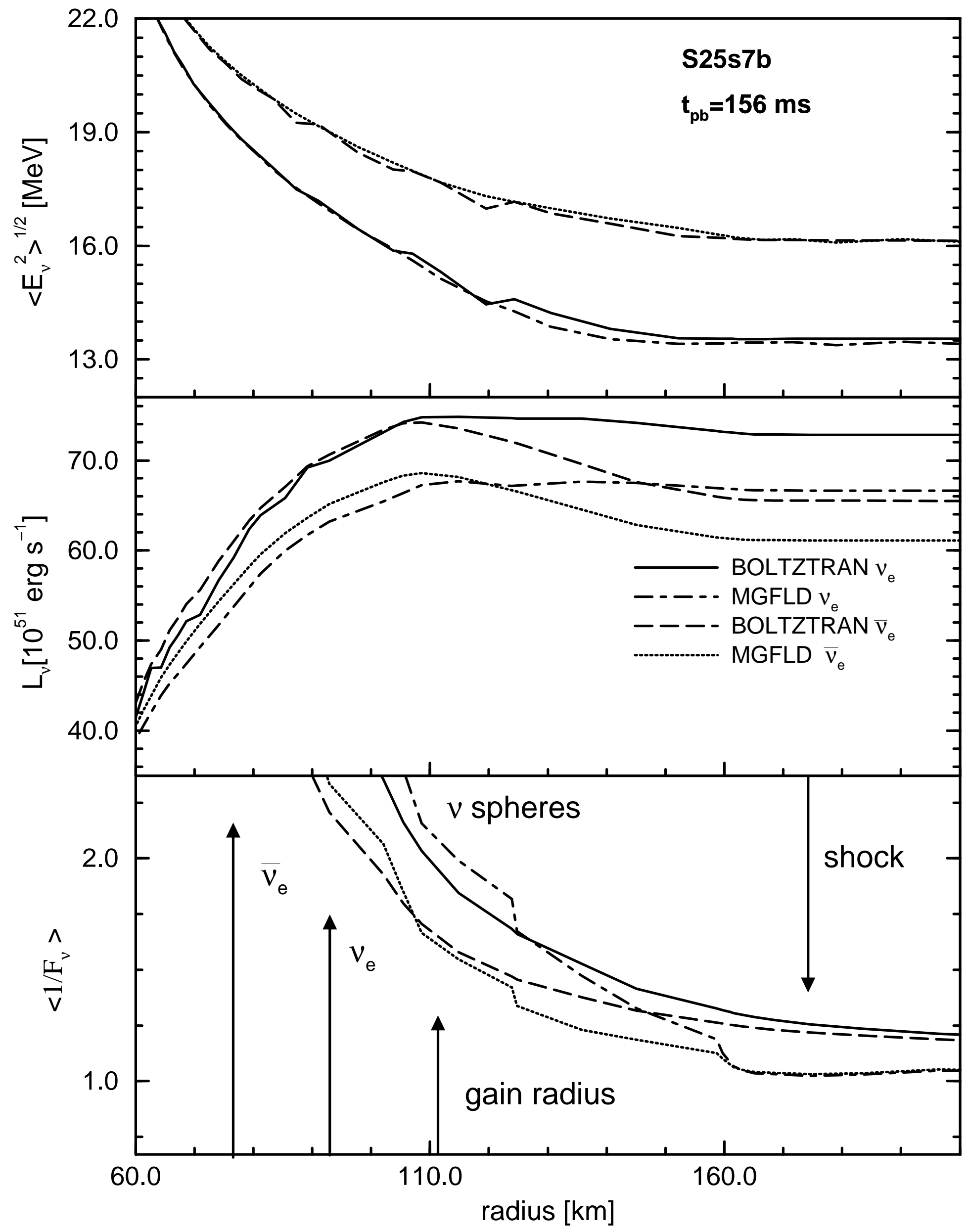

Figure 5 


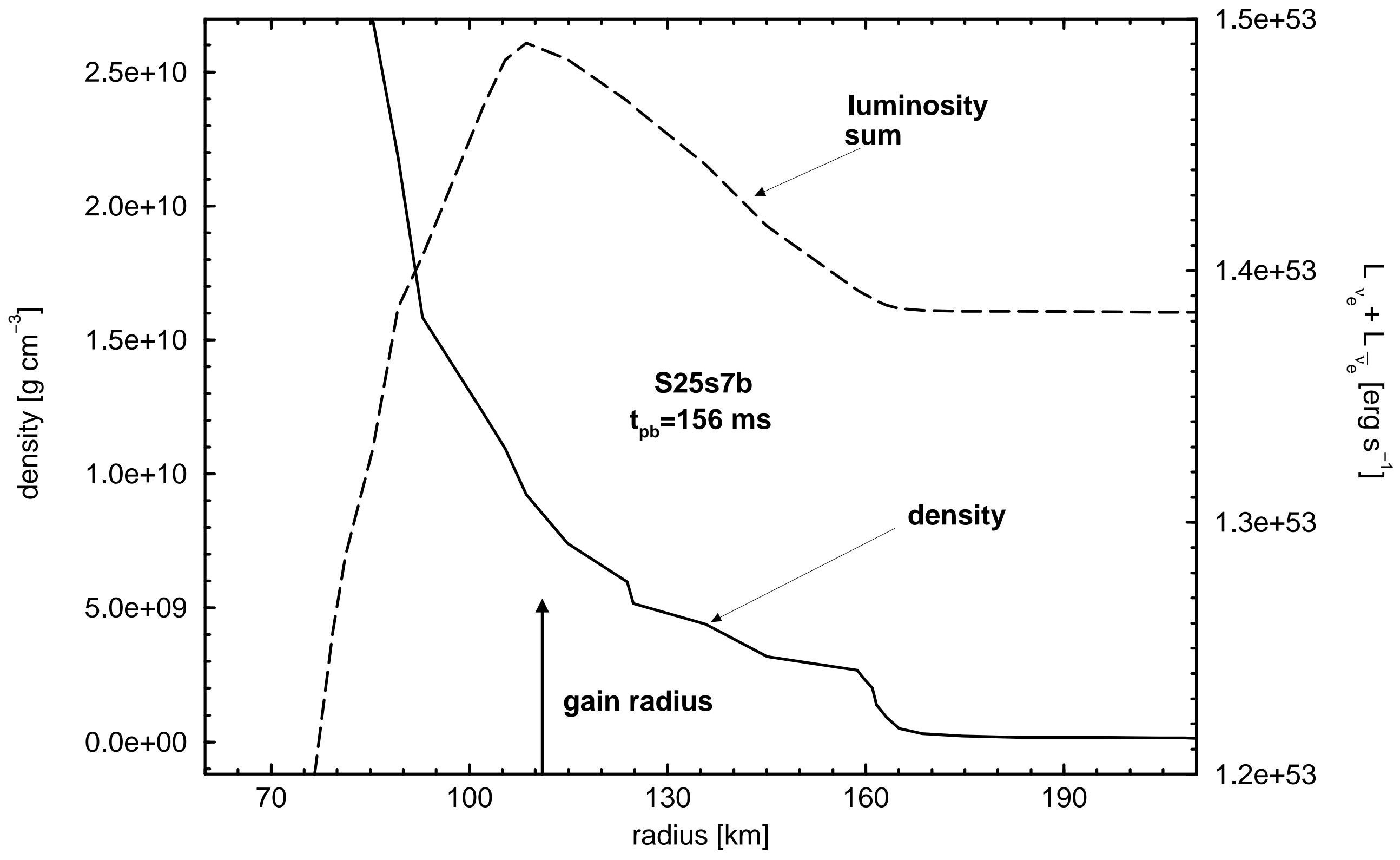

Figure 6 


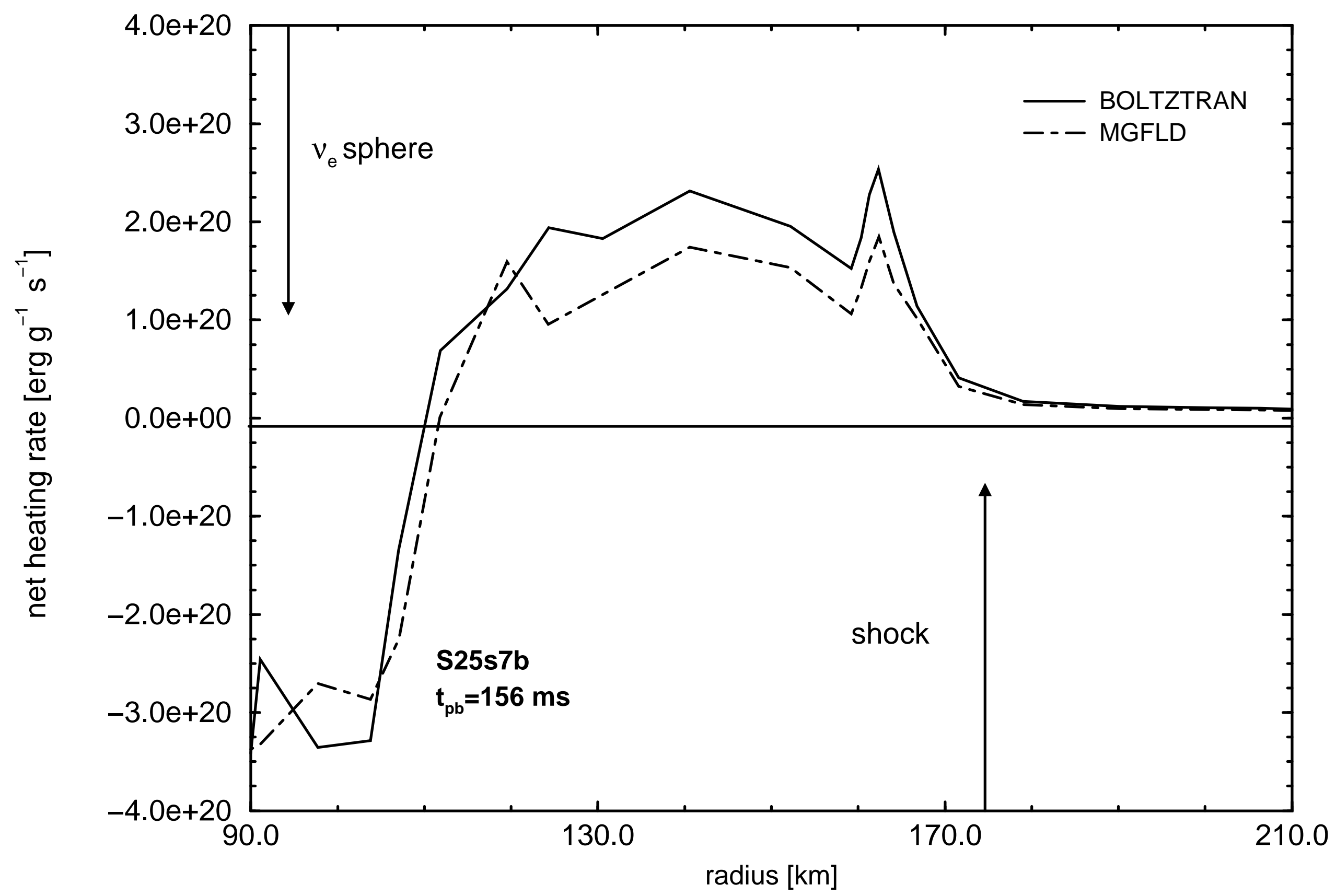

Figure 9 


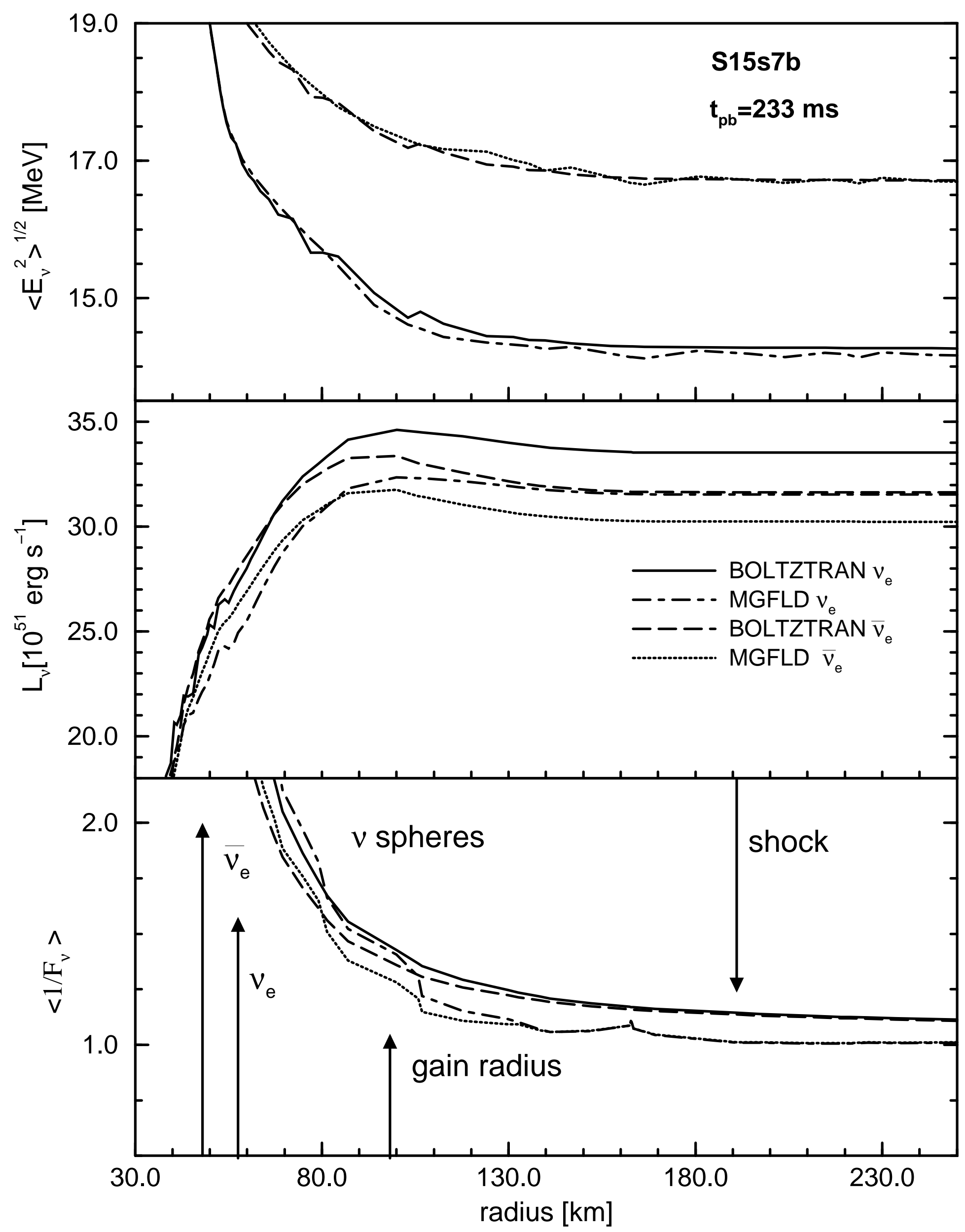

Figure 1 


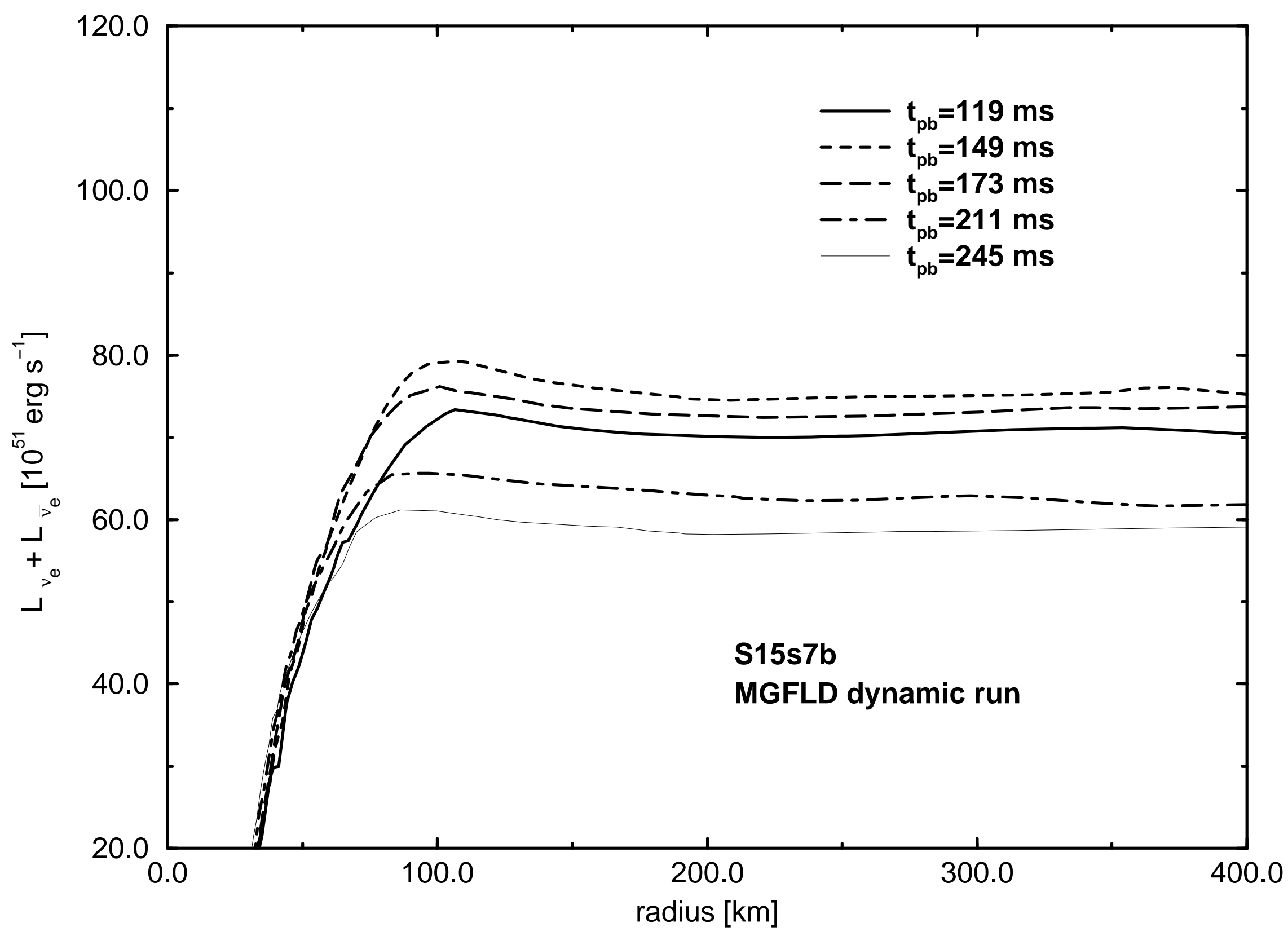

Figure 10 


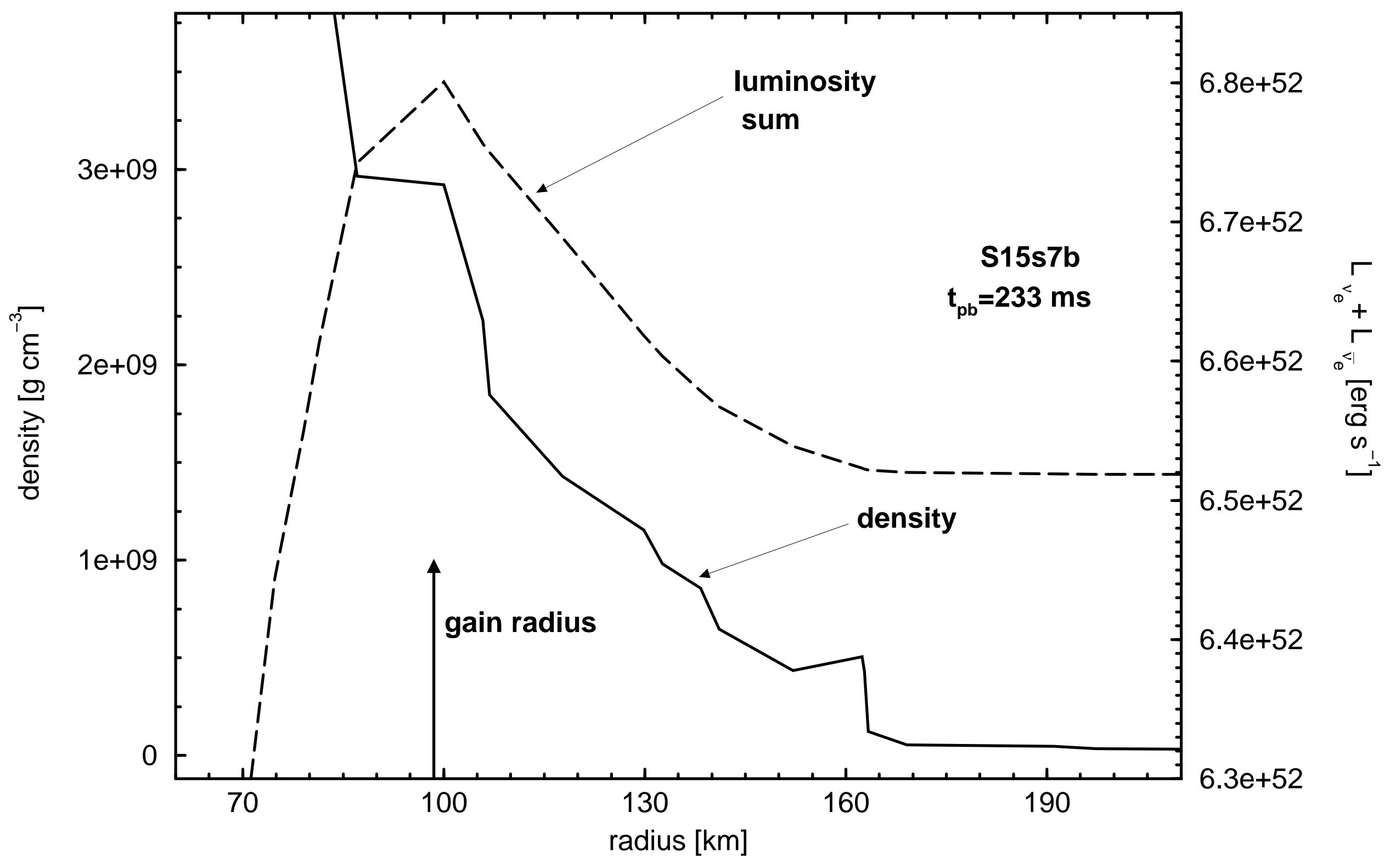

Figure 2 


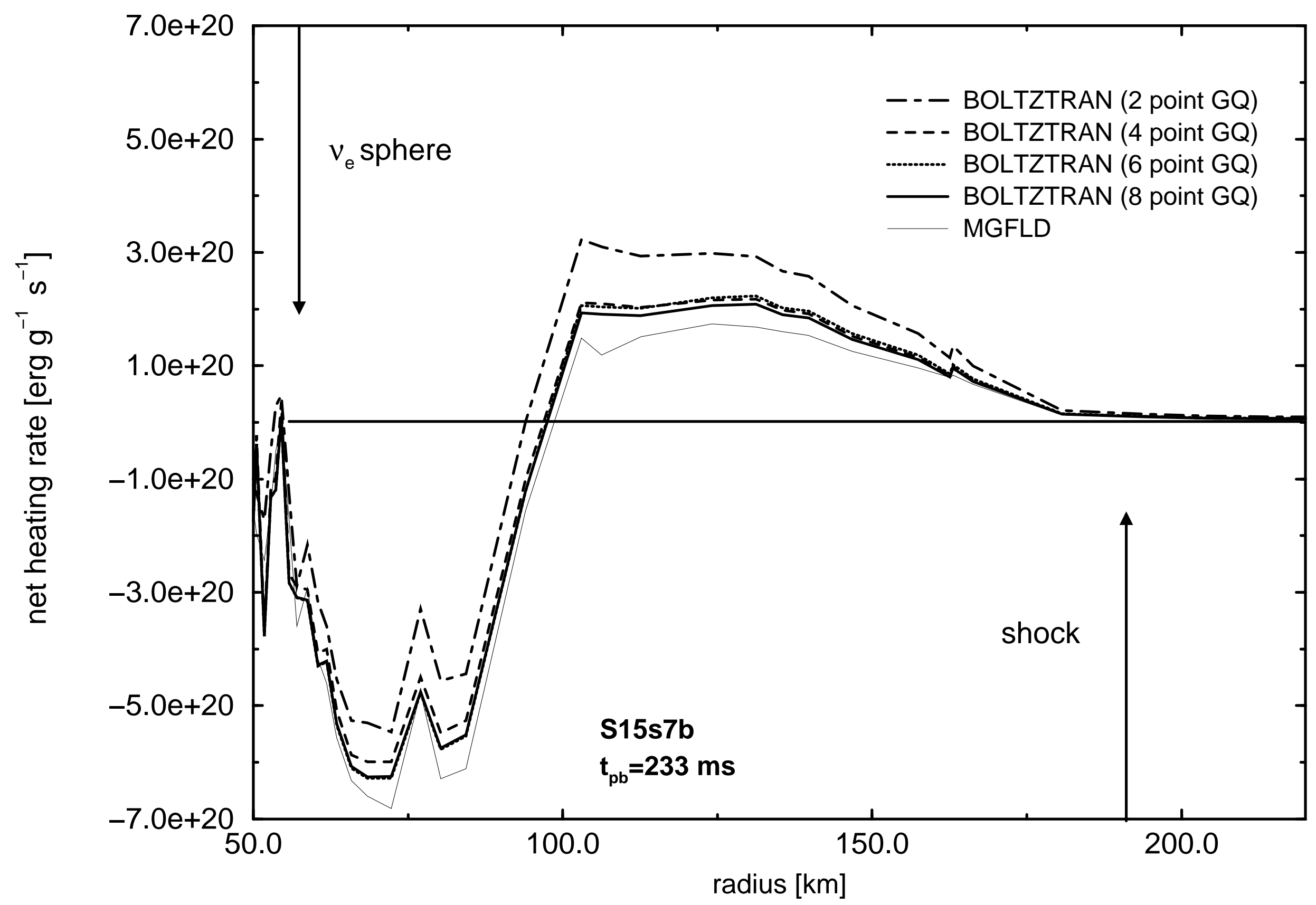

Figure 7 